\title{
Fratura em sinostose rádio-umeral: Descrição de dois casos clínicos*
}

\section{Fracture in Humeroradial Synostosis: Description of Two Clinical Cases}

\author{
Ricardo Kaempf de Oliveira ${ }^{1,2}$ João Pedro Farina Brunelli ${ }^{3 @} \quad$ Márcio Aurelio Aita ${ }^{4}$ \\ Pedro José Delgado Serrano 5
}

${ }^{1}$ Grupo de Cirurgia de Mão, Santa Casa de Misericórdia de Porto Alegre, Porto Alegre, RS, Brasil

${ }^{2}$ Hospital Mãe de Deus de Porto Alegre, Porto Alegre, RS, Brasil

${ }^{3}$ Hospital Santa Casa de São Paulo, São Paulo, SP, Brasil

4 Faculdade de Medicina do ABC, Santo André, SP, Brasil

5 Unidade de Cirurgia de Mão, Hospital Universitário Madrid

Montepríncipe, Universidade CEU San Pablo, Boadilla del Monte,

Madri, Espanha

Rev Bras Ortop

Endereço para correspondência Ricardo Kaempf de Oliveira, Rua Leopoldo Bier, 825, Conjunto 301, Porto Alegre, RS, 90620-100, Brasil (e-mail: ricardokaempf@gmail.com).

\section{Resumo}

Palavras-chave

- sinostose/cirurgia

- rádio/anormalidades

- anormalidades congênitas

- síndrome

- ulna/anormalidades

\section{Abstract}

Trabalho desenvolvido no Hospital Santa Casa de Porto Alegre e no Hospital Mãe de Deus, Porto Alegre, Brasil.

recebido

20 de Abril de 2020

aceito

06 de Julho de 2020
Sinostose é um termo genérico utilizado para indicar a união de dois ossos originalmente separados. No cotovelo, a sinostose rádio-umeral ou longitudinal causa importante incapacidade, que varia a depender da função da mão, da posição do cotovelo, da mobilidade das articulações adjacentes e da função do membro contralateral. Estima-se que um pouco mais de 150 pacientes foram descritos até hoje com essa deformidade, sendo mais frequente em portadores de deficiência de formação ulnar ou podendo fazer parte de síndromes como de Antley-Bixler e de Hermann. Devido à rigidez causada pela ausência da articulação do cotovelo, que resulta na formação de um osso mais longo com a fusão do úmero no rádio, presume-se que fratura nessa topografia não seja incomum. No entanto, pela raridade dessa patologia, tal lesão apresenta apenas duas descrições prévias na literatura. Relatamos os casos de dois pacientes com fratura do osso único formado pela sinostose entre o úmero e o rádio portadores de falha de formação ulnar do tipo IV de Bayne. Ambos os pacientes foram tratados de forma cirúrgica e evoluíram bem. Salientamos a necessidade do tratamento adequado para não comprometer as atividades da vida de um paciente já adaptado à deformidade, evitando piorar a função de um membro já alterado.

Synostosis is a generic term to indicate the union of two originally separated bones. At the elbow, humeroradial or longitudinal synostosis causes significant disability, which varies depending on hand function, elbow positioning, adjacent joints mobility and contralateral limb function. It is estimated that, to date, a little more than 150 patients have been described with this deformity, which is more common in subjects with 


\author{
Keywords \\ - synostosis/surgery \\ - radius/ \\ abnormalities \\ - congenital \\ abnormalities \\ - syndrome \\ - ulna/abnormalities.
}

deficient ulnar formation or affected by conditions such as Antley-Bixler and Hermann syndromes. The lack of the elbow joint, with the formation of a longer bone due to humerus-radius fusion, results in stiffness. As such, it is assumed that fractures in this topography are not uncommon. However, since synostosis is rare, this lesion was only described twice in the literature. We report two patients with a fracture of the single bone formed by a humeroradial synostosis and Bayne type-IV ulnar formation failure. Both patients were treated surgically with success. We emphasize the need for adequate treatment to not compromise the daily activities of patients who are adapted to their deformity, thus avoiding worsening the function of a previously affected limb.

\section{Introdução}

Sinostose é um termo genérico utilizado para indicar a união de dois ossos geralmente separados. No cotovelo, é importante diferenciar a sinostose rádio-ulnar proximal (SRUP) (transversa) da sinostose rádio-umeral (SRU) (longitudinal). A SRUP é menos rara e incapacitante, podendo ocorrer de forma isolada ou associada a algumas síndromes como a trissomia do 13 e do $21 .^{1}$ O paciente apresenta flexoextensão normal do cotovelo, com bloqueio da pronossupinação. Tal movimento pode ser compensado pela rotação do ombro e do punho, e, em pacientes selecionados, o tratamento cirúrgico apresenta bons resultados. ${ }^{1}$

Já a SRU é mais rara e ocorre em pacientes com falhas de formação longitudinal pós-axial (FFLPA), também chamadas de deficiência ulnar. Podem também fazer parte de síndromes como as de Antley-Bixler e de Hermann. ${ }^{2-7}$ Essa anomalia causa impossibilidade de flexão e extensão do cotovelo, gerando incapacidade variável, dependendo da função da mão, da posição do cotovelo, da mobilidade das articulações adjacentes e da função do membro contralateral. Os pacientes com deficiência ulnar associada à SRU são classificados como tipo IV por Bayne, e o envolvimento bilateral dos membros superiores não é raro (20\%), assim como a associação com outras deformidades esqueléticas como fêmur curto congênito, escoliose congênita, displasia do desenvolvimento do quadril, focomielia, hemimelia fibular e pé torto congênito. ${ }^{8-10}$ O tratamento indicado no caso desses pacientes é para melhora da função da mão e do seu posicionamento, principalmente para aqueles que apresentam a clássica posição de "mão no flanco", provocada pela rotação interna exagerada da extremidade superior, fazendo com que a mão fique rodada em
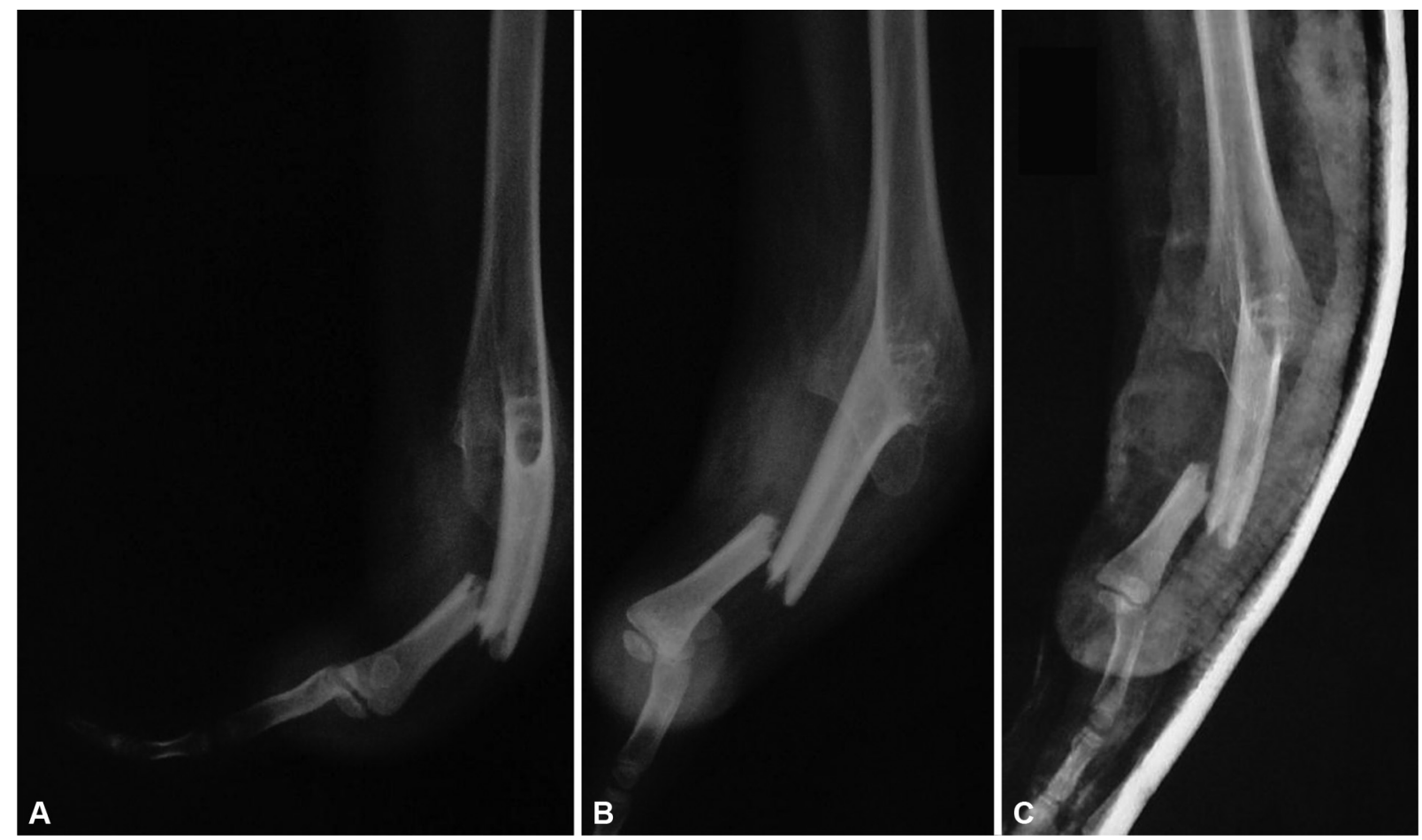

Fig. 1 Menino de 11 anos de idade com sinostose radioumeral sofreu queda ao solo e trauma no membro superior direito. As radiografias revelaram fratura do osso único formado pela SRU, logo distal ao local da sinostose (A), com angulação e encurtamento ósseo mesmo após tentativa de redução (B e C). 
direção à região glútea. ${ }^{1,8}$ A tentativa de cirurgia para recuperar a mobilidade no local da sinostose é, em geral, um fracasso. ${ }^{1,4}$

Devido à rigidez causada pela ausência da articulação do cotovelo, que resulta na formação de um osso mais longo com a fusão do úmero no rádio, presume-se que fratura nessa topografia não seja incomum. No entanto, pela raridade dessa patologia, tal lesão apresenta apenas duas descrições prévias na literatura. ${ }^{2,3}$

Relatamos os casos de dois pacientes com fratura do osso único formado pela sinostose úmero-radial portadores de mão torta ulnar (MTU) do tipo IV de Bayne. Salientamos a necessidade do tratamento adequado para não comprometer as atividades da vida de um paciente já adaptado à deformidade, evitando piorar a função de um membro já alterado.

\section{Relatos dos casos}

Tratamos um menino de 11 anos de idade, portador de grave e isolada malformação congênita dos membros superiores. Ele é o filho único de uma família sem história de alterações congênitas. Mãe refere não ter tido intercorrências na gestação, como doenças ou exposição à radiação, medicações, álcool ou drogas. Também negou história de casamentos consanguíneos na família. Ele já estava em acompanhamento desde o nascimento em nosso serviço de cirurgia de mão pediátrica. Apresenta o diagnóstico de FFLPA bilateral, com SRU (tipo IV de Bayne), apenas um dedo na mão direita, e agenesia dos dedos com sindactilia dos segundo e terceiro dedos da mão esquerda (tipo III de Bayne), já operada anteriormente. Fomos chamados ao pronto atendimento
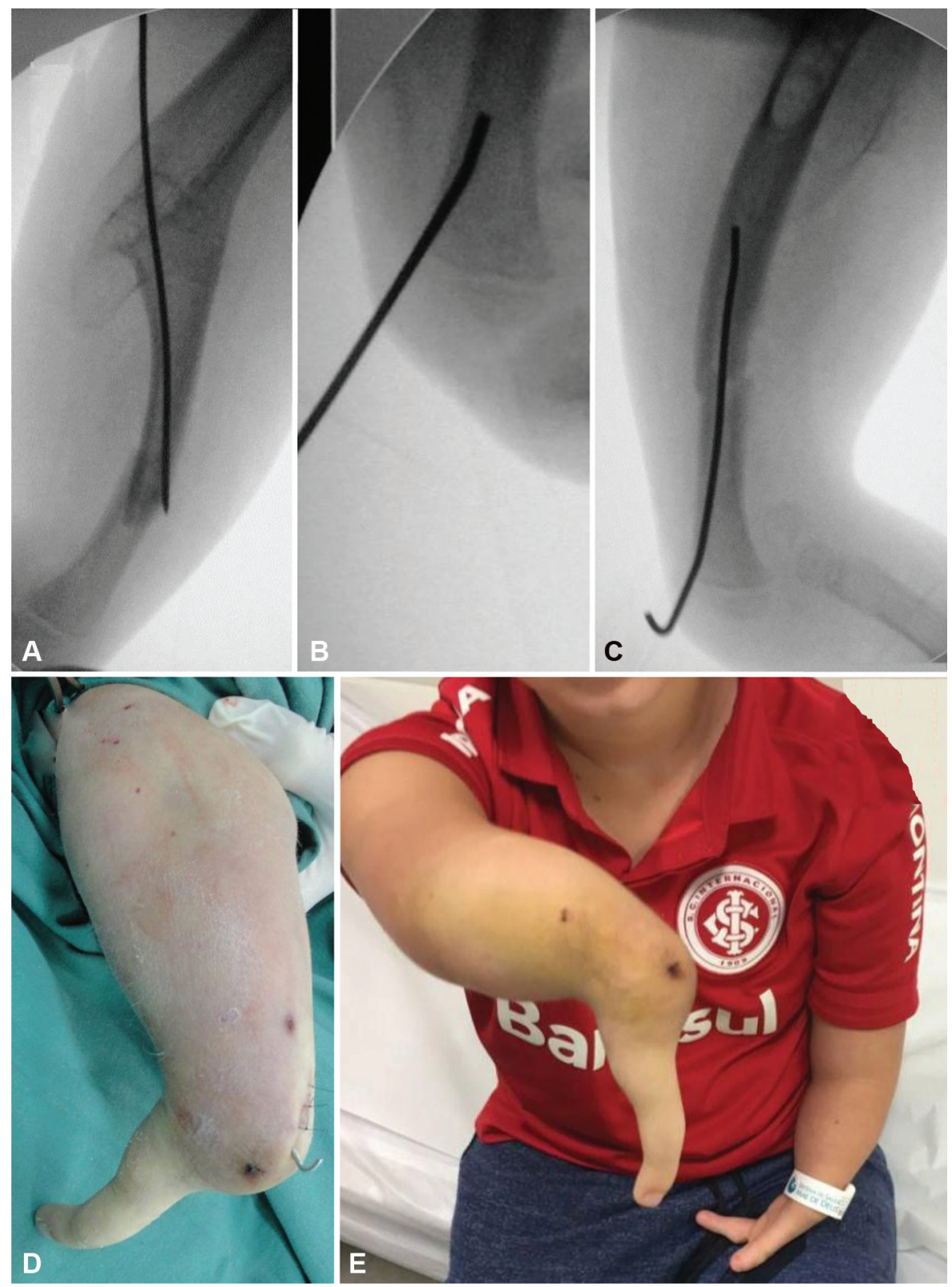

Fig. 2 Tentativa inicial, sem sucesso, de fixação da fratura com fio intramedulular anterógrado através do local da sinostose (A). Realizada fixação intramedular de forma retrógrada e percutânea (B, C e D). O paciente foi acompanhado em consultas mensais pelos primeiros 6 meses e com 3 anos de pós-operatório, o paciente não apresentava dor e com função semelhante à antes do acidente (E). 
para avaliar o paciente, que apresentava dor e deformidade no braço direito após queda de bicicleta. No exame, evidenciava-se deformidade e angulação no terço inferior do membro superior, sem déficit vascular ou neurológico, além das alterações já presentes ao nascimento. As radiografias iniciais revelaram fratura do osso único formado pela SRU, imediatamente distal ao sítio da sinostose (- Figura 1). Foi então confeccionada uma tala gessada axilopalmar, com tentativa de redução da fratura. Devido ao considerável desvio da fratura, foi indicado tratamento cirúrgico, realizado com anestesia geral. Inicialmente tentou-se fixação da fratura com fio intramedulular anterógrado. Porém, pelas alterações anatômicas locais, não foi possível que encontrássemos o canal medular encavilhamento. Optou-se, na sequência, pela fixação intramedular de forma retrógrada percutânea. Após confirmação radioscópica da redução da fratura, o paciente foi imobilizado com tala gessada. Nos dias seguintes, o paciente não apresentou qualquer complicação relacionada ao procedimento, como lesão nervosa ou vascular ( - Figura 2). Foram realizadas consultas semanais com controle radiográfico e trocas de talas gessadas. Com 20 dias de pósoperatório, a tala gessada foi retirada, e foi indicado o uso de tipoia e estimulada a realização de movimentos leves do punho e ombro. Os fios foram retirados com 8 semanas de evolução, quando as radiografias já evidenciavam sinais de consolidação da fratura. O paciente foi acompanhado em consultas mensais pelos primeiros 6 meses, sendo depois avaliado semestralmente. Foi liberado a praticar atividade física e esportes de contato com 4 meses de evolução. Numa avaliação tardia, com 3 anos de pós-operatório, o paciente não apresentava dor ou déficit funcional. As radiografias mostraram a consolidação completa da fratura, sem apresentar quaisquer complicações.
O segundo paciente tratado pela nossa equipe, do sexo masculino e com 62 anos de idade, foi admitido com o diagnóstico de FFLPA isolada no lado direito, com sinostose radio-umeral (tipo IV de Bayen) e agenesia do polegar. Ele é o segundo filho de uma família sem história de alterações congênitas, com mãe (já falecida) sem intercorrências na gestação. Ele chegou ao nosso hospital após queda de escada e trauma no membro superior direito. No exame, apresentava deformidade e angulação no terço superior do membro superior, sem déficit vascular ou neurológico. Radiografias evidenciaram fratura do osso único formado pela SRU, proximal à sinostose. Também foi evidenciada osteoartrite do ombro e do punho (-Figura 3). No primeiro atendimento foi confeccionada de forma provisória uma tala gessada axilopalmar, com alinhamento da fratura. Como tratamento definitivo foi optado pela fixação com placa, com uso de bloqueio de plexo braquial. A abordagem utilizada equivale à abordagem anterolateral padrão para úmeros anatomicamente normais. A única alteração anatômica relevante notada no procedimento foi o tamanho umeral, consideravelmente hipoplásico. 0 paciente não apresentou complicações precoces ou tardias relacionadas ao procedimento. $\mathrm{O}$ paciente não foi imobilizado no pós operatório, optando-se apenas pelo uso de tipoia. Previamente ao procedimento, foi oferecida a possibilidade de correção das deformidades em extensão do cotovelo e rotação interna extrema, o que foi negado pelo mesmo por considerar-se bem adaptado às alterações, com membro funcional. Foram realizadas consultas semanais, e com 6 semanas de evolução as radiografias evidenciavam sinais de consolidação da fratura. 0 paciente foi acompanhado em consultas mensais pelos primeiros 6 meses, sendo depois avaliado semestralmente. Foi liberado a praticar atividades da vida diária e esportes com 4 meses de
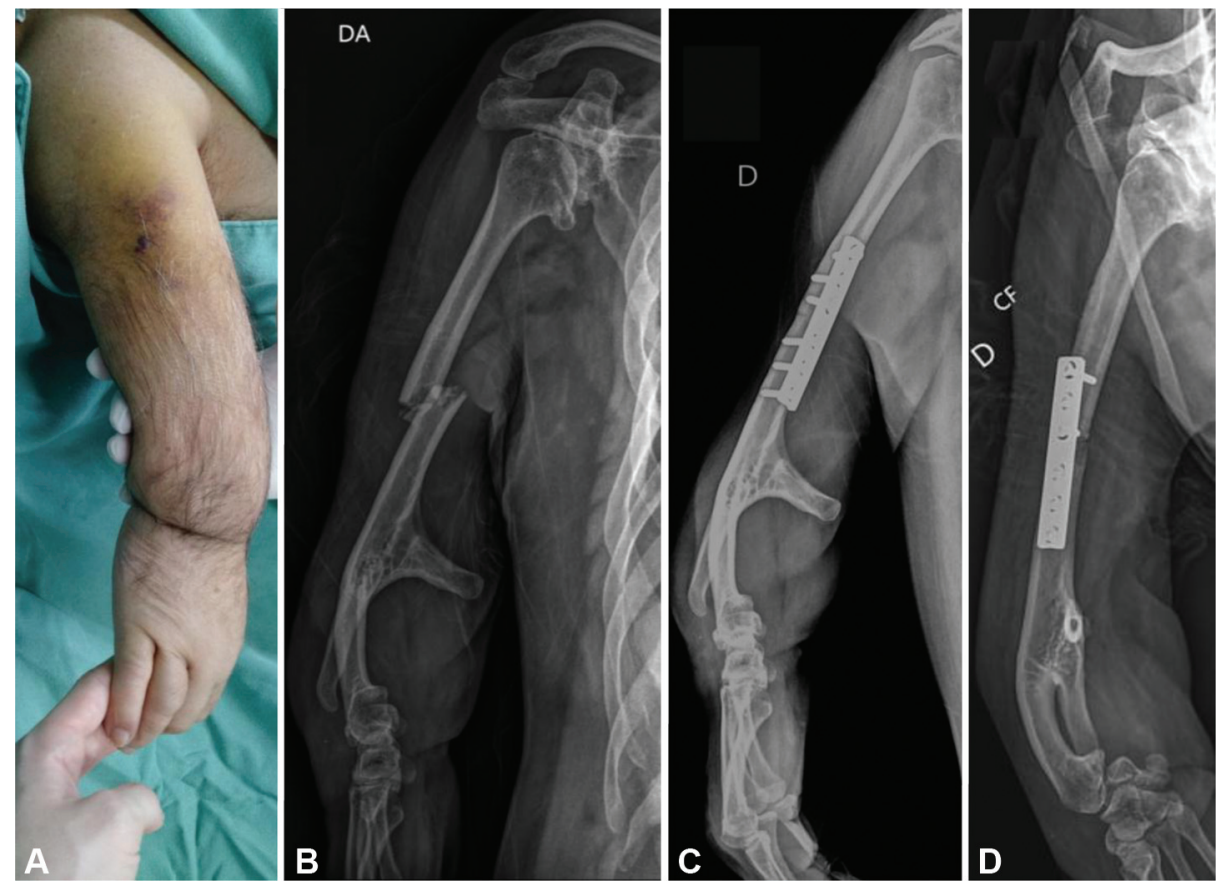

Fig. 3 Paciente de 62 anos com sinostose radioumeral sofreu queda de escada e trauma no membro superior direito, apresentando deformidade e dor no local (A). Radiografias comprovam fratura proximal a sinostose e artrose degenerativa do ombro e punho (B). O tratamento definitivo foi feito com a fixação da fratura com placa e parafusos (C e D). 


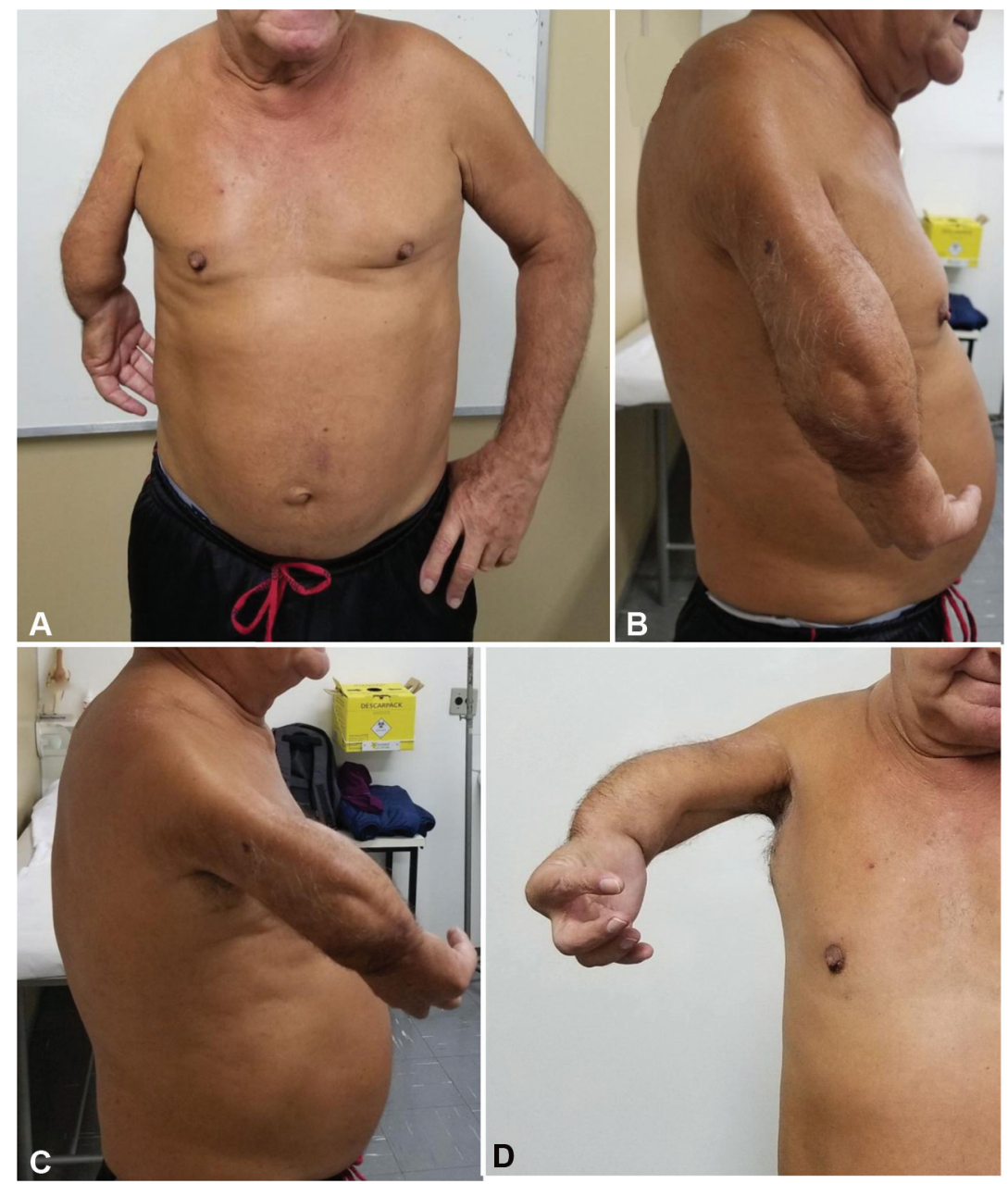

Fig. 4 Na avaliação com dois anos de evolução, o paciente apresentava o membro estável, sem dor e com função igual a relata antes da fratura (A, B, C e D).

evolução. Numa avaliação tardia com 2 anos de pós-operatório, o paciente não apresentava dor ou déficit funcional (-Figura 4).

\section{Discussão}

A incidência de fratura do osso único formado pela SRU não deveria ser uma lesão rara. A presença de um osso mais longo, decorrente da ausência da articulação do cotovelo, predispõe mecanicamente o membro a esta ocorrência. Porém, devido à raridade de pacientes com SRU, existem apenas dois pacientes descritos na literatura com fratura do membro anormal. Ambos os relatos enfocam muito mais a doença de base do paciente, o contexto da síndrome e as alterações na face, pouco valorizando a lesão óssea e o tratamento realizado.

A deformidade do cotovelo em paciente com FFLPA é tão característica que uma das primeiras classificações ${ }^{10}$ baseouse na presença de sinostose do cotovelo e de luxação da cabeça do rádio. Após isso, as classificações tenderam a se voltar mais para a presença da ulna e as deformidades na mão, como as de Ogden, Riordan, Swanson e Miller apud Cole e Manske. ${ }^{10}$ Atualmente a mais aceita é a de Bayne modificada, baseada na de Ogden, ${ }^{10}$ que divide as alterações em quatro tipos e inclui as deformidades do cotovelo e do antebraço ${ }^{1,10}$ ( - Tabela 1).
Tabela 1 Classificação de Bayne baseada na de Ogden, que divide as falhas de formação longitudinal pós-axial do membro superior em quatro tipos, conforme a gravidade e o acometimento do cotovelo.

\begin{tabular}{l}
\hline $\begin{array}{l}\text { Classificação de Bayne para falhas de formação long- } \\
\text { itudinal pós-axial }\end{array}$ \\
\hline $\begin{array}{l}\text { Tipo I. Ulna hipoplásica, com epífises proximais e distais } \\
\text { presentes. }\end{array}$ \\
\hline $\begin{array}{l}\text { Tipo II. Ausência parcial da ulna, com falha na região distal } \\
\text { (tipo mais frequente). }\end{array}$ \\
\hline Tipo III. Ausência total da ulna. \\
\hline $\begin{array}{l}\text { Tipo IV. Ausência da ulna com sinostose radioumeral } \\
\text { (tipo mais raro). }\end{array}$
\end{tabular}

A incidência da SRU é desconhecida. Em 1955, Card e Strachman ${ }^{11}$ reportaram o $35^{\circ}$ na literatura. Anos após, Swanson et al. ${ }^{9}$ e Buck-Gramcko apud McIntyre et al. ${ }^{5}$ publicaram séries com 47 e 20 pacientes, respectivamente. Atualmente, estima-se que haja 150 casos reportados na literatura. ${ }^{6}$ Nas séries de FFLPA, a sua presença varia de 20 a 53\%, lembrando que a incidência de FFLPA é 4 a 10 vezes mais baixa que a da pré-axial (radial). ${ }^{1,10}$ Em pacientes com boa posição da mão e com o cotovelo em semi-flexão, não há indicação de cirurgia. Os resultados obtidos com a tentativa 
cirúrgica de obter movimento no local sinostose são invariavelmente pobres ${ }^{1,4}$ Tabrizi et al. $^{4}$ relataram a completa recidiva da sinostose após a tentativa de ressecção da barra óssea e interposição de gordura para melhora a posicionamento da mão . Alguns pacientes com SRU apresentam o membro superior em rotação interna exagerada, permanecendo a mão rodada para as costas, a chamada "mão no flanco". Tal posição é extremamente incapacitante e Miller e James recomendam a osteotomia rotatória externa do úmero para esses pacientes. ${ }^{1,8}$

McIntyre et al. ${ }^{5}$ classificam as SRU em dois tipos. O tipo I é o mais comum, sendo três vezes mais frequente, e é uma deformidade esporádica e associada à FFLPA e hipoplasias dos dedos, com o cotovelo em extensão. Já no tipo II, a sinostose tem um caráter familiar, estando associada a síndromes, com o cotovelo fixo em flexão e não apresentando alterações na mão. Nossos dois pacientes reportados são do tipo I, e pensamos que o cotovelo em extensão aumentou o risco de lesão e fraturas pela maior suscetibilidade a trauma e pelo aumento do braço de alavanca gerado por um osso longo e reto.

Pensamos que a presença de outras alterações congênitas esqueléticas associadas a SRU, como escápula dupla ou bífida, podem ser uma tentativa de aumentar a mobilidade do membro, compensando a rigidez do cotovelo. Nesses pacientes o risco de fratura no local da sinostose seria menor. Seguindo esse raciocínio, pensamos que um dos fatores causais da fratura do nosso segundo paciente, além da severidade do trauma, seja a presença de artrose nas articulações do punho e ombro, provocando uma maior rigidez de todo o membro superior.

Os pacientes com SRU geralmente apresentam outras alterações esqueléticas e, como a maioria dos pacientes com deformidades congênitas, apresentam algum grau de adaptação. ${ }^{1}$ Assim sendo, qualquer mudança da anatomia e da posição do membro, principalmente em pacientes adultos, pode dificultá-los nas atividades da vida diária. Em ambos os pacientes por nós tratados preferimos o tratamento cirúrgico, refazendo a anatomia original e evitando uma maior deformidade e encurtamento do membro, além de encurtar o período de imobilização. Para pacientes que apresentam grave rotação interna do membro e que sofram fratura, o tratamento cirúrgico pode ser uma boa oportunidade de correção da deformidade rotacional.

Conflito de Interesses

Os autores declaram não haver nenhum conflito de interesses.

\section{Referências}

1 Kozin SH. Congenital differences about the elbow. Hand Clin 2009; 25(02):277-291

2 McCall AA, Kirsch CF, Ishiyama G, Ishiyama A. Otologic findings in Antley-Bixler syndrome: a clinical and radiologic case report. Int J Pediatr Otorhinolaryngol 2007;71(07):1139-1143

3 Hosalkar HS, Shah HS, Gujar PS, Shaw BA. The Antley-Bixler syndrome: two new cases. J Postgrad Med 2001;47(04):252-255

4 Tabrizi A, Afshar A, Aidenlou A. A case of failure to correct humerus-radius synostosis and thumb hypoplasia. J Hand Surg Eur Vol 2018;43(02):211-213

5 McIntyre JD, Brooks A, Benson MK. Humeroradial synostosis and the multiple synostosis syndrome: case report. J Pediatr Orthop B 2003;12(03):192-196

6 Nema S, Vyas G, Sirsikar A, Bhoj PK. Congenital humeroradial synostosis: a case report. Malays Orthop J 2012;6(SupplA):41-42. Doi: 10.5704/MOJ.1211.010

7 Pfeiffer RA, Braun-Quentin C. Genetic nosology and counseling of humeroradial synostosis. Genet Couns 1994;5(03):269-274

8 Miller JK, Wenner SM, Kruger LM. Ulnar deficiency. J Hand Surg Am 1986;11(06):822-829

9 Swanson AB, Tada K, Yonenobu K. Ulnar ray deficiency: its various manifestations. J Hand Surg Am 1984;9(05):658-664

10 Cole RJ, Manske PR. Classification of ulnar deficiency according to the thumb and first web. J Hand Surg Am 1997;22(03):479-488

11 Card RY, Strachman J. Congenital ankylosis of the elbow. J Pediatr 1955;46(01):81-85 